Parte II - Dados Censitários em Contexto

\title{
Capítulo 7 - Parturição em Mulheres Indígenas e Não Indígenas do Brasil: o número de filhos relatados depende de quem responde às perguntas do censo nacional?
}

\author{
Ricardo Ventura Santos \\ João Luiz Bastos \\ Oswaldo Gonçalves Cruz \\ Luciene Aparecida Ferreira de Barros Longo \\ Nancy May Flowers \\ Nilza de Oliveira Martins Pereira
}

\section{SciELO Books / SciELO Livros / SciELO Libros}

SANTOS, R.V., BASTOS, J.L., LONGO, L.A.F.B., FLOWERS, N.M., and PEREIRA, N.O.M. Parturição em Mulheres Indígenas e Não Indígenas do Brasil: o número de filhos relatados depende de quem responde às perguntas do censo nacional?. In: SANTOS, R.V., GUIMARÃES, B.N., CAMPOS, M.B., and AZEVEDO, M.M.A., comps. Entre Demografia e Antropologia: povos indígenas no Brasil [online]. Rio de Janeiro: Editora FIOCRUZ, 2019, pp. 157-178. Saúde dos povos Indígenas collection. ISBN: 978-655708-013-9. https://doi.org/10.7476/9786557080139.0008.

All the contents of this work, except where otherwise noted, is licensed under a Creative Commons Attribution 4.0 International license.

Todo o conteúdo deste trabalho, exceto quando houver ressalva, é publicado sob a licença Creative Commons Atribição 4.0. 


\section{Parturição em Mulheres Indígenas e Não Indígenas do Brasil: o número de filhos relatados depende de quem responde às perguntas do censo nacional?*}

Ricardo Ventura Santos

João Luiz Bastos

Oswaldo Gonçalves Cruz

Luciene Aparecida Ferreira de Barros Longo

Nancy May Flowers

Nilza de Oliveira Martins Pereira

Em razão das trajetórias históricas distintas, das variadas formas de classificação e da falta de dados estatísticos confiáveis, estimar o tamanho das populações indígenas ao redor do mundo se impõe como uma tarefa de considerável complexidade (Axelsson G Sköld, 2011; Paho, 2006; UN, 2009). Em meio ao reconhecimento dessa complexidade, observa-se uma preocupação crescente com os dados de natureza demográfica e de saúde desses grupos étnicos específicos, os quais tendem a apresentar piores condições de vida do que as sociedades nacionais que compartilham do mesmo território. Com o objetivo de descrever as condições de vida e avaliar a magnitude das desigualdades em suas dimensões demográficas e de saúde, agências internacionais têm encorajado países das mais variadas regiões do planeta a incluir questões voltadas para as condições sociodemográficas dos povos indígenas em suas pesquisas censitárias de abrangência nacional (Paho, 2006; UN, 2009).

Estima-se que há mais de 400 diferentes grupos indígenas na América Latina e no Caribe, com uma população total de cerca de 50 milhões de indivíduos (McSweeney G Arps, 2005; Montenegro G Stephens, 2006). Enquanto mais de 40\% da população da Bolívia, Equador, Guatemala e Peru se declara indígena, na Argentina, Brasil, Costa Rica e Uruguai, os povos indígenas perfazem menos de $1 \%$ do contingente populacional total de cada um desses países (Montenegro G Stephens, 2006). De modo semelhante ao que ocorre em diversas regiões do mundo, os povos indígenas da América Latina estão submetidos a piores condições de vida, menores níveis de escolaridade, maiores taxas de mortalidade, bem como a outras condições sociais e de saúde desfavoráveis, se comparados com suas respectivas sociedades nacionais (Coimbra G Santos, 2004; Incayawar G Maldonado-Bouchard, 2009; McSweeney G Arps, 2005; Montenegro G Stephens, 2006; Paho, 2006).

Em paralelo, a maior parte dos países latino-americanos tem incluído, e efetivamente expandido, o número de questões sobre os povos indígenas em suas pesquisas censitárias nacionais nas últimas décadas. A quantidade de perguntas, bem como o seu conteúdo específico, varia de país para país, embora um aspecto 
comum tenha sido a iniciativa de coletar dados sobre o tamanho das populações indígenas e suas filiações étnicas. O caso brasileiro é ilustrativo dessa tendência. Tendo sido incluída pela primeira vez em 1991, a categoria "indígena" passou a figurar entre as opções de resposta para o quesito cor/raça dos censos conduzidos no país desde então. Além disso, no Censo de 2010, os respondentes que optaram pela categoria "indígena" do quesito cor/raça também foram indagados a respeito da filiação étnica e das línguas nativas faladas em seus domicílios (IBGE, 2005, 2012; Santos G Teixeira, 2011).

É inquestionável que a inclusão de características demográficas dos povos indígenas nos censos nacionais de países latino-americanos e de outras regiões representa um expressivo avanço em direção a uma melhor compreensão das suas condições de vida e, portanto, à implementação e avaliação dos impactos de uma ampla variedade de políticas públicas (Del Popolo, 2008; Del Popolo et al., 2011; Montenegro G Stephens, 2006; UN, 2009). Contudo, é também importante reconhecer que, em graus variáveis, as características socioculturais das sociedades indígenas, em conjunto com determinados procedimentos metodológicos empregados nos censos nacionais, podem influenciar o modo como indígenas respondem às questões apresentadas pelos recenseadores (Axelsson G Sköld, 2011). No caso brasileiro, por exemplo, diversas comunidades indígenas contam com apenas alguns (ou nenhum) indivíduos fluentes em português, a língua oficialmente utilizada nas entrevistas censitárias (IBGE, 2012). Outros aspectos culturais que podem afetar o relato de informações aos recenseadores dizem respeito aos sistemas numéricos, os quais são distintos do sistema decimal comumente empregado nas análises demográficas oficiais, especialmente nas comunidades que retêm suas línguas nativas. Isso também se estende aos sistemas indígenas de atribuição de idade, os quais raramente se baseiam na data de nascimento (Pagliaro, Azevedo G Santos, 2005), como o faz o censo brasileiro. Dessa forma, sempre que possível, a influência desses fatores deve ser considerada na apreciação dos dados censitários referentes aos povos indígenas.

Tendo-se a parturição como variável de interesse primário, o objetivo deste capítulo é investigar se os padrões de resposta ao censo diferem entre indígenas e não indígenas, quando essa informação é provida diretamente pela mulher ou por um respondente proxy (morador ou não do próprio domicílio). Com esse propósito, utilizamos dados sobre o número de filhos relatados por mulheres indígenas e não indígenas de duas regiões brasileiras, especificamente o Norte e o Nordeste. Tais regiões foram selecionadas por diferirem marcadamente em termos de características antropológicas e históricas de seus povos indígenas, como, por exemplo, uma proporção expressivamente maior de indígenas falando suas línguas nativas no Norte do que no Nordeste, entre outras importantes distinções. Espera-se que nossa análise indique os fatores que podem ter influenciado parte dos resultados do 
Censo Demográfico 2010, subsidiando, portanto, o planejamento e a implementação de futuras iniciativas voltadas para a coleta de dados em comunidades indígenas e outros segmentos populacionais. Em uma dimensão mais ampla, nosso trabalho contribui para uma reflexão crítica acerca da maneira como os dados sobre minorias étnicas indígenas, que estão entre as populações mais marginalizadas do mundo em termos de saúde e direitos humanos, têm sidos coletados pelos Estados nacionais.

\section{Fontes de dados e análises realizadas}

No Brasil, os censos demográficos nacionais têm sido conduzidos desde a segunda metade do século XIX. A partir de 1940, o Instituto Brasileiro de Geografia e Estatística (IBGE) se tornou o órgão federal responsável pelos censos, que são conduzidos em intervalos regulares de dez anos (Gonçalves, 1995; IBGE, 2006; Nobles, 2000).

O Censo Demográfico 2010 empregou dois tipos de questionário. O questionário básico foi aplicado ao universo da população brasileira, enquanto o questionário da amostra foi administrado em um subgrupo de respondentes derivado do próprio censo. O primeiro abrangeu uma quantidade limitada de características dos domicílios investigados (em sua maior parte, relacionadas à composição domiciliar e aspectos ligados ao saneamento), bem como de seus próprios residentes, incluindo, por exemplo, idade, sexo, cor/raça, alfabetização e renda. Todas as perguntas do questionário básico fizeram parte do questionário da amostra, o qual também abordou itens sobre ocupação, fecundidade e migração, entre outros. Detalhes de cada um dos questionários podem ser conferidos na página <http://censo2010.ibge.gov.br>.

Em cada domicílio, um único indivíduo foi entrevistado (geralmente, um adulto identificado como o chefe do domicílio em questão), que deveria prover informações sobre si mesmo e sobre todos os demais moradores. Segundo os procedimentos metodológicos do Censo 2010, um não morador do domicílio também poderia, caso necessário, prover informações sobre seus membros. Com o objetivo de identificar esses diferentes respondentes, a base de dados do Censo 2010 incluiu a variável denominada "tipo de respondente" ou "marca", com as seguintes possibilidades de classificação: 1) a informação foi dada diretamente pelo entrevistado, isto é, foi autorrelatada; 2) a informação sobre algum membro do domicílio foi fornecida por outro morador; 3) a informação sobre algum morador foi provida por um não morador (frequentemente, uma trabalhadora doméstica que estava no local, por ocasião da visita do recenseador, quando nenhum residente estava presente). Num total de cerca de 190 milhões de brasileiros recenseados, 4,2\% deles, em média, tiveram suas informações fornecidas por não moradores de seus próprios domicílios. 
O Censo Demográfico 2010 foi o primeiro na história do país a conduzir as entrevistas com o auxílio de dispositivos do tipo Personal digital assistant, um aparelho eletrônico móvel que armazena e organiza informações, evitando o uso de questionários impressos. Além disso, diferentemente do que ocorreu em edições prévias, o Censo 2010 foi o primeiro a incluir o quesito cor/raça no questionário básico, aplicando-o à totalidade da população do país. Entre outros avanços importantes na coleta de dados, esse censo também coletou informação sobre filiação étnica para todos os declarados indígenas (IBGE, 2012), línguas indígenas faladas no domicílio e residência em reservas indígenas reconhecidas pelo governo federal. O quesito cor/raça dividiu a população em cinco categorias: branca, preta, amarela, parda e indígena (IBGE, 2013). Os respondentes poderiam ignorar essa pergunta, situação na qual seriam classificados com cor/raça "desconhecida". Tais indivíduos somaram menos de $0,1 \%$ do total da população e foram excluídos das análises do presente trabalho.

Os recenseadores que participaram da coleta de dados de 2010 não receberam treinamento específico para conduzir entrevistas em áreas indígenas (IBGE, 2013). Entretanto, o material desenvolvido pelo IBGE incluiu orientações específicas destinadas a uma melhor compreensão dos povos indígenas e seus domicílios, com foco nos padrões de habitação, práticas religiosas e atividades econômicas locais, tendo em vista que esses tópicos seriam abordados em parte das perguntas do questionário (IBGE, 2010a, 2013). Em regiões do país com maiores concentrações de terras indígenas (TIs), o IBGE também disponibilizou folhetos aos entrevistadores com informações gerais sobre os povos indígenas, preparados em colaboração com antropólogos e demógrafos vinculados ao Grupo de Trabalho Demografia dos Povos Indígenas no Brasil da Associação Brasileira de Estudos Populacionais. A motivação principal para a confecção dos folhetos foi sensibilizar os entrevistadores para os distintos padrões culturais e sociais com os quais poderiam ter contato nos territórios indígenas, incluindo o encontro de indivíduos completamente despidos até a expectativa de que se apresentassem aos líderes locais previamente à condução das entrevistas do censo.

Para desenvolver o presente estudo, um subconjunto de variáveis de nível individual do Censo 2010 foi convertido para o formato Stata (programa estatístico para uso em computadores pessoais), abarcando as seguintes informações: estrutura amostral (incluindo os setores censitários individuais como unidades primárias de amostragem e grupos contíguos de setores censitários como estratos), pesos amostrais, situação urbana/rural, número de moradores e quantidade de cômodos do domicílio, cor/raça, escolaridade, situação conjugal e idade. A principal variável de interesse - parturição ou a contagem de todos os filhos relatados por uma mulher ao longo de sua vida reprodutiva - também integrou o conjunto de variáveis e foi definida pelo número de filhos nascidos vivos até julho de 2010, tal como informada aos recenseadores. 
As análises foram restritas às mulheres acima dos 10 anos de idade residindo na região Norte ou Nordeste do país. Tais regiões foram selecionadas para comparação, pois os povos indígenas que nelas residem apresentam diferenças sócio-históricas de particular importância para este trabalho. Enquanto os indígenas do Nordeste foram os primeiros a sofrer os impactos da chegada dos europeus no século XVI, os do Norte, região que apresenta grande sobreposição com a Amazônia, passaram a interagir com não indígenas num período mais recente, especialmente a partir do século XX (Hemming, 1987; Ricardo G Ricardo, 2011). Como resultado dessas trajetórias históricas complexas e, ao mesmo tempo, distintas, uma maior quantidade de grupos indígenas do Norte ainda fala suas línguas nativas, reside em reservas e mantém sistemas econômicos baseados em práticas de subsistência (Hemming, 1987; Ricardo G Ricardo, 2011). Dados os vários séculos de presença não indígena no Nordeste, os povos indígenas que lá residem não apenas perderam a maior parte de seus territórios tradicionais, mas também passaram por transformações culturais que culminaram na extinção de suas línguas nativas. Enquanto 55,2\% dos declarados indígenas do Norte relataram falar alguma língua nativa em seus domicílios, tal percentual foi de 13,6\% no Nordeste (IBGE, 2012). A região Norte também conta com o maior contingente populacional indígena do país (342.836 indivíduos de um total de 896.917 em todo o Brasil). Por sua vez, a população do Nordeste inclui um número expressivo de indígenas, 126.597 indivíduos, sendo que a maior parte deles vive fora de áreas de reserva (IBGE, 2012). Por fim, a região Norte dispõe do maior percentual $(82,0 \%)$ de grupos indígenas em áreas rurais (IBGE, 2012).

O primeiro passo da análise estatística consistiu na descrição de cada grupo de cor/raça, em ambas as regiões investigadas, de acordo com a situação urbana/rural, escolaridade e idade. Em seguida, dois importantes parâmetros deste estudo, isto é, (1) a frequência de entrevistas segundo cada tipo de respondente e (2) a parturição, foram estimados - de acordo com a situação urbano/rural, cor/ raça, idade (em faixas etárias até 60 anos ou mais) e escolaridade (desde analfabeto até ensino superior completo, incluindo uma categoria para escolaridade não registrada) - para o Norte e para o Nordeste, separadamente. A parturição também foi estimada de acordo com o tipo de respondente. A associação entre cor/raça e parturição, bem como o impacto do tipo de respondente sobre essa relação foram avaliados com modelos de regressão binomial negativa inflacionada de zeros (BNIZ), estratificando-se para região (Norte e Nordeste) e situação urbano/rural.

Como forma de avaliar se a associação entre cor/raça e parturição diferiu em decorrência do tipo de respondente, um termo de interação entre essas variáveis foi acrescentado às equações de regressão. A inclusão desse termo procurou testar a hipótese de que mulheres indígenas teriam menor chance de, relativamente às não indígenas, relatar suas informações diretamente aos recenseadores devido à menor fluência em português e a dinâmicas de gênero específicas (expressas, por 
exemplo, na tendência de homens a interagirem mais frequentemente com visitantes não indígenas em seus territórios). Isso aumentaria a chance de que as informações sobre a história reprodutiva das mulheres fossem fornecidas por diversos indivíduos, tais como, outros membros do domicílio ou, mesmo, algum não morador das residências abordadas. Os critérios de informação de Bayes e de Akaike indicaram que os modelos com o termo de interação apresentaram melhor ajuste aos dados.

As covariáveis incluídas nos modelos foram: idade, escolaridade, número de cômodos do domicílio (número que variou de 1 a 30) e total de moradores da residência (variável numérica discreta com amplitude de 1 a 56). Todas as variáveis independentes foram mantidas nos modelos, não importando o seu impacto nas estimativas de ajuste. Objetivando facilitar a interpretação, apenas os coeficientes para contagem de filhos são discutidos neste capítulo. A média de filhos predita pelos modelos, juntamente com seus intervalos de confiança de 95\% (IC95\%), de acordo com cor/raça e tipo de respondente, também foi estimada, estratificando-se para região e situação urbano/rural, após ajuste para as demais covariáveis. Todas as análises levaram em consideração a amostragem complexa, bem como os pesos amostrais, tal como recomendado pelo IBGE. ${ }^{1}$

\section{Achados do estudo}

A análise abrangeu 6.290.856 mulheres acima de 10 anos de idade na região Norte do Brasil, e 22.817.444 no Nordeste (Tabela 1). A proporção de mulheres declaradas brancas, pretas, amarelas, pardas e indígenas em cada região, respectivamente, foi de: Norte $-24,0 \%, 6,2 \%, 1,3 \%, 66,9 \%$ e 1,6\%; Nordeste $29,6 \%, 9,4 \%, 1,4 \%, 59,2 \%$ e $0,4 \%$. Entre os respondentes da região Norte, 76,3\% das mulheres declaradas pardas e $83,2 \%$ das amarelas residiam em áreas urbanas, ao passo que a maioria das mulheres indígenas $(75,7 \%)$ residia em áreas rurais. Entretanto, o percentual de mulheres indígenas residindo em situação urbana foi consideravelmente maior no Nordeste $(57,7 \%)$. A proporção de não indígenas em áreas urbanas no Nordeste variou entre 73,0\% (pardas) e 79,8\% (brancas), valores ligeiramente mais baixos do que o observado no Norte.

A Tabela 1 demonstra igualmente que, entre todos os grupos investigados nas duas regiões, mulheres indígenas apresentaram os menores níveis de escolaridade. No Norte e no Nordeste, $82,4 \%$ e 64,3\% eram analfabetas ou tinham apenas ensino fundamental incompleto, respectivamente. Mulheres declaradas pretas e pardas ocuparam posição intermediária em termos de escolarização, ao passo que as classificadas como amarelas e brancas apresentaram maiores níveis de educação formal. As indígenas também tenderam a ser mais jovens, concentrando-se nas duas primeiras categorias de faixa etária: 10-14 e 15-19 anos. 
Em ambas as regiões estudadas, pouco mais da metade das entrevistadas responderam aos recenseadores diretamente (51,2\% no Norte e $54,4 \%$ no Nordeste) (Tabela 2). Em outras palavras, 45,6\% a 48,8\% das entrevistas foram conduzidas por respondentes proxy, os quais poderiam ser tanto outro morador quanto um não morador do próprio domicílio. O percentual de mulheres que respondeu diretamente aos entrevistadores foi um pouco maior nas áreas rurais, especialmente no Norte (55,6\%), o mesmo ocorreu com mulheres mais velhas, especificamente aquelas entre 50 e 59 anos. A associação entre tipo de respondente e escolaridade não seguiu um padrão consistente e claro. Mulheres indígenas do Norte tiveram a maior porcentagem de entrevistas realizadas com um não morador de seus domicílios (12,7\% no total; $15,0 \%$ e 3,0\% em áreas rurais e urbanas, respectivamente). É digno de nota que os demais grupos de cor/raça da região Norte apresentaram, relativamente, frequências bastante baixas de entrevistas realizadas com não moradores: brancas $-4,0 \%$, pretas $-2,9 \%$, amarelas $-2,5 \%$ e pardas $-3,0 \%$. Em comparação com a região Norte, as frequências de respostas dadas pelas próprias mulheres, por outro morador ou não morador do domicílio variaram menos entre indígenas e não indígenas do Nordeste.

A Tabela 3 contém as médias de parturição, conforme situação urbana/ rural, escolaridade, cor/raça, idade e tipo de respondente no Norte e no Nordeste. Essas contagens médias foram ligeiramente mais elevadas no Nordeste (2,15 filhos) do que no Norte (2,08 filhos). A parturição foi maior nas áreas rurais, em grupos de menor escolaridade e, conforme esperado, entre as mulheres mais velhas. As mulheres indígenas apresentaram a maior média de filhos, sendo imediatamente seguidas pelas pretas. Mulheres declaradas brancas e amarelas tiveram as menores médias de parturição. Independentemente de cor/raça, as médias foram consideravelmente mais elevadas quando essa informação foi provida pela própria mulher - tais valores foram 93,5\%-101,4\% maiores, quando comparados com aqueles informados por outros moradores dos domicílios, e 15,6\%-21,7\% maiores, em contraste com o relatado por um não morador.

Quando a parturição foi estimada no contexto de modelos de regressão BNIZ, ajustados para todas as covariáveis, as mulheres indígenas contaram com médias 18\%-39\% maiores, relativamente às brancas (Tabela 4). Ao se considerarem todos os grupos de cor/raça em conjunto, observou-se que as médias de parturição foram mais baixas quando informadas por outros moradores do domicílio, em comparação com a situação em que as próprias mulheres forneceram tal informação: as diferenças variaram entre 1,0\% (Norte rural) a 7,0\% (Nordeste urbano). O termo de interação entre cor/raça e tipo de respondente revelou um aspecto central desta análise: mulheres indígenas do Norte rural, cujas respostas ao censo foram dadas por um não morador apresentaram uma contagem de filhos $16 \%$ menor $(0,84$, IC95\% 0,75-0,93), se comparadas com as brancas que proveram essa informação 
diretamente aos entrevistadores. Essa foi a maior diferença detectada entre todos os grupos comparados, tanto no Norte quanto no Nordeste. Apesar de as diferenças para as indígenas e amarelas do Norte urbano serem ainda maiores $(0,78$ e 0,82, respectivamente), tais resultados não foram estatisticamente significativos. No que se refere à parturição para indígenas, suas estimativas foram 10,0\% (0,90, IC95\% 0,82-0,99) e 7,0\% (0,93, IC95\% 0,88-0,99) menores em áreas urbanas e rurais, respectivamente, quando a contagem de filhos foi informada por outro morador do domicílio (essas comparações têm mulheres brancas, que responderam diretamente ao recenseador, como categoria de referência). Houve uma associação negativa entre parturição e escolaridade, bem como uma relação positiva com idade. Nem o total de cômodos, nem o número de moradores dos domicílios estiveram associados com a parturição.

As estimativas de parturição preditas pelos modelos de regressão, ilustradas nos Gráficos 1 e 2, sugerem que esse parâmetro é afetado pelo tipo de respondente em todos os grupos de cor/raça, tanto nas áreas urbanas quanto nas rurais das duas regiões investigadas. Em geral, o tipo de respondente apresentou um impacto semelhante nas médias de parturição em todos os grupos de cor/raça do Nordeste, com as estimativas mais baixas derivando de outros moradores dos domicílios, enquanto as mais altas foram informadas diretamente pelas mulheres (Gráfico1). Na maior parte das vezes, as médias não diferiram quando comparados outros moradores e não moradores dos domicílios, tendo em vista a sobreposição dos intervalos de confiança. Em termos comparativos, o impacto do tipo de respondente sobre as contagens médias de filhos foi maior para as mulheres indígenas do Norte, especialmente nas áreas rurais (Gráfico 2). Se, por um lado, a parturição média foi de 3,1 (IC95\% 3,0-3,2) nas áreas rurais do Norte (quando essa informação foi fornecida pela própria mulher), por outro, a mesma estimativa ficou em torno de 2,4 (IC95\% 2,3-2,6) para outro morador e não morador dos domicílios investigados. No Norte urbano, as médias de parturição foram um pouco maiores quando estimadas para as mulheres que proveram essa informação diretamente aos entrevistadores $(2,6$, IC95\% 2,5-2,8), relativamente à situação em que tal dado foi fornecido por outro morador (2,1, IC95\% 1,9-2,2) ou não morador do domicílio $(2,0$, IC95\% $1,4-2,5)$. 
Tabela 1 - Distribuição relativa da amostra por situação urbana/rural, escolaridade e idade, de acordo com cor/raça, estratificada por região. Regiões Norte e Nordeste - Brasil - 2010

\begin{tabular}{|c|c|c|c|c|c|c|c|c|c|c|}
\hline \multirow{3}{*}{ Variável } & \multicolumn{10}{|c|}{ Cor/raça - \% } \\
\hline & \multicolumn{5}{|c|}{ Norte } & \multicolumn{5}{|c|}{ Nordeste } \\
\hline & Branca & Preta & Amarela & Parda & Indígena & Branca & Preta & Amarela & Parda & Indígena \\
\hline \multicolumn{11}{|l|}{ Situação urbana/rural } \\
\hline Urbana & 82,5 & 76,7 & 83,2 & 76,3 & 24,3 & 79,8 & 78,8 & 77,7 & 73,0 & 57,7 \\
\hline Rural & 17,5 & 23,3 & 16,8 & 23,7 & 75,7 & 20,2 & 21,2 & 22,3 & 27,0 & 42,3 \\
\hline \multicolumn{11}{|l|}{ Escolaridade } \\
\hline $\begin{array}{l}\text { Analfabeto/ensino } \\
\text { primário incompleto }\end{array}$ & 45,5 & 57,8 & 44,7 & 54,6 & 82,4 & 48,5 & 58,4 & 50,9 & 58,4 & 64,3 \\
\hline $\begin{array}{l}\text { Ensino fundamental } \\
\text { completo/ ensino médio } \\
\text { incompleto }\end{array}$ & 17,0 & 16,2 & 19,2 & 17,5 & 8,7 & 15,6 & 15,2 & 17,1 & 16,1 & 15,2 \\
\hline $\begin{array}{l}\text { Ensino médio completo/ } \\
\text { ensino superior } \\
\text { incompleto }\end{array}$ & 29,7 & 20,5 & 28,1 & 22,4 & 7,2 & 25,4 & 22,0 & 25,2 & 20,6 & 16,4 \\
\hline $\begin{array}{l}\text { Ensino superior } \\
\text { completo }\end{array}$ & 10,1 & 5,0 & 7,1 & 4,8 & 1,2 & 10,0 & 3,9 & 6,2 & 4,4 & 3,7 \\
\hline Não informada & 0,7 & 0,5 & 0,9 & 0,7 & 0,5 & 0,5 & 0,5 & 0,6 & 0,5 & 0,4 \\
\hline \multicolumn{11}{|l|}{ Idade (anos) } \\
\hline $10-14$ & 12,3 & 11,2 & 11,1 & 14,4 & 19,2 & 10,4 & 9,1 & 10,0 & 12,1 & 13,2 \\
\hline $15-19$ & 12,3 & 11,3 & 13,0 & 13,3 & 15,6 & 10,5 & 9,9 & 11,8 & 11,7 & 12,5 \\
\hline $20-29$ & 23,4 & 24,0 & 28,6 & 24,3 & 23,2 & 21,2 & 22,2 & 25,2 & 22,1 & 20,4 \\
\hline $30-39$ & 19,2 & 19,1 & 21,4 & 18,8 & 16,2 & 17,6 & 18,7 & 19,4 & 17,8 & 17,6 \\
\hline $40-49$ & 13,7 & 13,7 & 12,3 & 12,8 & 10,0 & 14,6 & 14,9 & 13,8 & 14,1 & 13,8 \\
\hline $50-59$ & 9,1 & 9,9 & 6,5 & 8,4 & 6,6 & 10,4 & 11,1 & 8,8 & 10,1 & 9,2 \\
\hline $60+$ & 9,1 & 10,8 & 7,1 & 8,0 & 9,2 & 15,3 & 14,1 & 11,0 & 12,1 & 13,3 \\
\hline Total (n) & $\begin{array}{r}100,0 \\
(1.505 .492)\end{array}$ & $\begin{array}{r}100,0 \\
(390.747)\end{array}$ & $\begin{array}{r}100,0 \\
(81.798)\end{array}$ & $\begin{array}{r}100,0 \\
(4.210 .557)\end{array}$ & $\begin{array}{r}100,0 \\
(102.262)\end{array}$ & $\begin{array}{r}100,0 \\
(6.768 .722)\end{array}$ & $\begin{array}{r}100,0 \\
(2.151 .841)\end{array}$ & $\begin{array}{r}100,0 \\
(310.191)\end{array}$ & $\begin{array}{r}100,0 \\
(13.501 .871)\end{array}$ & $\begin{array}{r}100,0 \\
(84.819)\end{array}$ \\
\hline
\end{tabular}


Tabela 2 - Frequência relativa de tipo de respondente do Censo 2010, conforme situação urbana/rural, escolaridade, cor/raça e idade, estratificada por região. Regiões Norte e Nordeste - Brasil - 2010

\begin{tabular}{c|c|c|c|c|c|c}
\multirow{2}{*}{ Variável } & \multicolumn{5}{|c}{ Tipo de respondente } \\
\cline { 2 - 6 } & \multicolumn{3}{|c|}{ Norte } \\
\cline { 2 - 6 } & $\begin{array}{c}\text { A } \\
\text { própria } \\
\text { mulher }\end{array}$ & $\begin{array}{c}\text { Outro } \\
\text { morador do } \\
\text { domicílio }\end{array}$ & $\begin{array}{c}\text { Não } \\
\text { morador do } \\
\text { domicílio }\end{array}$ & $\begin{array}{c}\text { A } \\
\text { própria } \\
\text { mulher }\end{array}$ & $\begin{array}{c}\text { Outro } \\
\text { morador do } \\
\text { domicílio }\end{array}$ & $\begin{array}{c}\text { Não } \\
\text { morador do } \\
\text { domicílio }\end{array}$ \\
\hline
\end{tabular}

Situação urbana/rural

\begin{tabular}{|c|c|c|c|c|c|c|}
\hline Urbana & 49,9 & 46,8 & 3,3 & 53,4 & 43,0 & 3,6 \\
\hline Rural & 55,6 & 40,8 & 3,6 & 57,6 & 39,3 & 3,1 \\
\hline \multicolumn{7}{|l|}{ Escolaridade } \\
\hline $\begin{array}{l}\text { Analfabeto/ensino } \\
\text { fundamental incompleto }\end{array}$ & 50,1 & 46,5 & 3,4 & 55,4 & 41,1 & 3,5 \\
\hline $\begin{array}{l}\text { Ensino fundamental } \\
\text { completo/ensino médio } \\
\text { incompleto }\end{array}$ & 52,2 & 45,0 & 2,8 & 52,8 & 44,3 & 2,9 \\
\hline $\begin{array}{l}\text { Ensino médio completo/ } \\
\text { ensino superior } \\
\text { incompleto }\end{array}$ & 53,1 & 43,2 & 3,7 & 53,5 & 42,8 & 3,7 \\
\hline Ensino superior completo & 52,3 & 43,7 & 4,0 & 54,3 & 42,1 & 3,6 \\
\hline Não informada & 36,4 & 61,4 & 2,2 & 35,8 & 61,2 & 3,0 \\
\hline \multicolumn{7}{|l|}{ Cor/raça } \\
\hline Branca & 45,4 & 50,6 & 4,0 & 49,6 & 46,3 & 4,1 \\
\hline Preta & 57,3 & 39,8 & 2,9 & 59,3 & 37,4 & 3,3 \\
\hline Amarela & 62,1 & 35,4 & 2,5 & 63,4 & 34,3 & 2,3 \\
\hline Parda & 52,7 & 44,3 & 3,0 & 55,9 & 40,9 & 3,2 \\
\hline Indígena & 41,9 & 45,4 & 12,7 & 55,2 & 41,0 & 3,8 \\
\hline \multicolumn{7}{|l|}{ Idade (anos) } \\
\hline $10-14$ & 16,9 & 80,4 & 2,7 & 17,5 & 80,2 & 2,3 \\
\hline $15-19$ & 34,4 & 63,0 & 2,6 & 34,5 & 63,2 & 2,3 \\
\hline $20-29$ & 55,4 & 40,9 & 3,7 & 54,5 & 41,9 & 3,6 \\
\hline $30-39$ & 62,8 & 33,8 & 3,4 & 64,9 & 31,8 & 3,4 \\
\hline $40-49$ & 63,6 & 33,4 & 3,0 & 66,2 & 30,8 & 3,0 \\
\hline $50-59$ & 65,2 & 31,2 & 3,6 & 69,0 & 27,6 & 3,4 \\
\hline $60+$ & 60,8 & 33,7 & 5,5 & 64,6 & 29,5 & 5,9 \\
\hline Total & 51,2 & 45,4 & 3,4 & 54,4 & 42,1 & 3,5 \\
\hline
\end{tabular}

Fonte: estimativas produzidas pelos próprios autores com base nos dados analisados. 
Tabela 3 - Número médio de filhos tidos (parturição) relatado ao Censo 2010 conforme situação urbana/rural, escolaridade, cor/raça, idade e tipo de respondente, estratificado por região. Regiões Norte e Nordeste - Brasil - 2010

\begin{tabular}{|c|c|c|}
\hline \multirow{2}{*}{ Variável } & \multicolumn{2}{|c|}{ Média de filhos - parturição (IC95\%) } \\
\hline & Norte & Nordeste \\
\hline \multicolumn{3}{|l|}{ Situação urbana/rural } \\
\hline Urbana & $1,95(1,94-1,96)$ & $1,98(1,98-1,99)$ \\
\hline Rural & $2,54(2,53-2,56)$ & $2,65(2,64-2,66)$ \\
\hline \multicolumn{3}{|l|}{ Escolaridade } \\
\hline Analfabeto/ensino primário incompleto & $2,65(2,64-2,66)$ & $2,90(2,89-2,90)$ \\
\hline $\begin{array}{l}\text { Ensino fundamental completo/ ensino médio } \\
\text { incompleto }\end{array}$ & $1,53(1,52-1,54)$ & $1,32(1,31-1,32)$ \\
\hline Ensino médio completo/ ensino superior incompleto & $1,43(1,42-1,44)$ & $1,15(1,14-1,15)$ \\
\hline Ensino superior completo & $1,39(1,38-1,41)$ & $1,26(1,25-1,27)$ \\
\hline Não informada & $0,69(0,64-0,74)$ & $0,53(0,51-0,55)$ \\
\hline \multicolumn{3}{|l|}{ Cor/raça } \\
\hline Branca & $1,90(1,89-1,92)^{*}$ & $2,02(2,01-2,02)^{*}$ \\
\hline Preta & $2,38(2,35-2,41)$ & $2,28(2,27-2,29)$ \\
\hline Amarela & $1,87(1,81-1,92)^{*}$ & $2,04(2,01-2,07)^{*}$ \\
\hline Parda & $2,12(2,11-2,13)$ & $2,19(2,19-2,20)$ \\
\hline Indígena & $2,46(2,40-2,51)$ & $2,52(2,45-2,58)$ \\
\hline \multicolumn{3}{|l|}{ Idade (anos) } \\
\hline $10-14$ & $0,01(0,01-0,01)$ & $0,01(0,01-0,01)$ \\
\hline $15-19$ & $0,23(0,22-0,23)$ & $0,16(0,16-0,17)$ \\
\hline $20-29$ & $1,26(1,25-1,26)$ & $0,97(0,97-0,97)$ \\
\hline $30-39$ & $2,42(2,41-2,43)$ & $2,03(2,03-2,04)$ \\
\hline $40-49$ & $3,23(3,22-3,25)$ & $2,82(2,81-2,83)$ \\
\hline $50-59$ & $4,25(4,22-4,28)$ & $3,91(3,89-3,92)$ \\
\hline $60+$ & $5,84(5,80-5,87)$ & $5,67(5,65-5,68)$ \\
\hline \multicolumn{3}{|l|}{ Tipo de respondente } \\
\hline A própria mulher & $2,69(2,68-2,70)$ & $2,74(2,74-2,75)$ \\
\hline Outro morador do domicílio & $1,39(1,38-1,40)$ & $1,36(1,35-1,36)$ \\
\hline Não morador do domicílio & $2,21(2,16-2,25)$ & $2,37(2,34-2,39)$ \\
\hline Total & $2,08(2,08-2,09)$ & $2,15(2,14-2,15)$ \\
\hline
\end{tabular}

Obs.: Intervalo de confiança (IC) de 95\%.

* Estas médias são estatisticamente diferentes das demais, dentro de cada uma das regiões estudadas ( $p<0,05$, de acordo com teste de heterogeneidade de Wald).

As médias globais para o Norte e o Nordeste são estatisticamente diferentes entre si ( $p<0,05$, conforme o teste de heterogeneidade de Wald).

Fonte: estimativas produzidas pelos próprios autores com base nos dados analisados. 
Tabela 4 - Regressão binomial negativa para estimar a associação entre cor/raça e parturição, estratificada por região e situação urbana/rural, ajustada para idade, escolaridade, total de cômodos do domicílio e número de moradores do domicílio. Regiões Norte e Nordeste - Brasil - 2010

\begin{tabular}{|c|c|c|c|c|}
\hline \multirow{3}{*}{ Variável } & \multicolumn{4}{|c|}{$\begin{array}{l}\text { Coeficiente de regressão para parturição } \\
\text { (intervalo de confiança de } 95 \% \text { ) }\end{array}$} \\
\hline & \multicolumn{2}{|c|}{ Norte } & \multicolumn{2}{|c|}{ Nordeste } \\
\hline & Urbana & Rural & Urbana & Rural \\
\hline \multicolumn{5}{|l|}{ Cor/raça } \\
\hline Branca & $1,00(-)$ & $1,00(-)$ & $1,00(-)$ & $1,00(-)$ \\
\hline Preta & $1,10(1,08-1,12) \uparrow$ & $1,15(1,12-1,19)$ व & $1,04(1,03-1,05)$ ฯ & $1,09(1,07-1,10)$ ฯ \\
\hline Amarela & $1,08(1,04-1,12)$ ฯ & $1,03(0,96-1,11)$ & $1,06(1,04-1,08)$ ฯ & $1,09(1,06-1,12) \uparrow$ \\
\hline Parda & $1,12(1,11-1,14)$ ฯ & $1,18(1,16-1,20)$ ฯ & $1,09(1,08-1,09)$ ฯ & $1,09(1,08-1,10) \pi$ \\
\hline Indígena & $1,39(1,31-1,47)$ ฯ & $1,34(1,29-1,39) \pi$ & $1,18(1,13-1,24)$ ฯ & $1,27(1,22-1,32) \pi$ \\
\hline \multicolumn{5}{|l|}{ Tipo de respondente } \\
\hline A própria mulher & $1,00(-)$ & $1,00(-)$ & $1,00(-)$ & $1,00(-)$ \\
\hline Outro morador do domicílio & $0,98(0,96-0,99)$ बा & $0,99(0,97-1,03)$ & $0,93(0,92-0,93) \rrbracket$ & $0,94(0,92-0,95) \pi$ \\
\hline Não morador do domicílio & $0,97(0,93-1,01)$ & $1,04(0,96-1,12)$ & $0,97(0,95-0,98) \rrbracket$ & $0,98(0,95-1,01)$ \\
\hline \multicolumn{5}{|c|}{ Cor/raça x Tipo de respondente (termo de interação)* } \\
\hline Preta, outro morador & $0,94(0,91-0,98) \pi$ & $0,97(0,92-1,02)$ & $0,95(0,94-0,97)$ ฯ & $0,98(0,95-1,01)$ \\
\hline Preta, não morador & $1,01(0,91-1,12)$ & $1,00(0,86-1,18)$ & $0,92(0,88-0,96)$ ฯ & $0,88(0,83-0,94)$ ๆ \\
\hline Amarela, outro morador & $0,90(0,83-0,97)$ ๆ & $0,94(0,83-1,07)$ & $0,96(0,92-1,00)$ & $0,97(0,92-1,04)$ \\
\hline Amarela, não morador & $0,78(0,59-1,04)$ & $0,98(0,77-1,26)$ & $0,90(0,80-1,01)$ & $1,02(0,88-1,18)$ \\
\hline Parda, outro morador & $0,93(0,91-0,95)$ ฯ & $0,93(0,90-0,96) \pi$ & $0,93(0,92-0,94)$ ฯ & $0,96(0,95-0,98)$ ฯ \\
\hline Parda, não morador & $0,96(0,92-1,01)$ & $0,90(0,83-0,98) \pi$ & $0,90(0,88-0,93)$ ๆ & $0,91(0,88-0,95)$ ฯ \\
\hline Indígena, outro morador & $0,90(0,82-0,99)$ ฯ & $0,93(0,88-0,99) \pi$ & $0,96(0,88-1,04)$ & $0,98(0,91-1,06)$ \\
\hline Indígena, não morador & $0,82(0,63-1,07)$ & $0,84(0,75-0,93) \pi$ & $0,99(0,84-1,17)$ & $0,91(0,78-1,06)$ \\
\hline \multicolumn{5}{|l|}{ Escolaridade } \\
\hline $\begin{array}{l}\text { Analfabeto/ensino primário } \\
\text { incompleto }\end{array}$ & $1,00(-)$ & $1,00(-)$ & $1,00(-)$ & $1,00(-)$ \\
\hline $\begin{array}{l}\text { Ensino primário completo/ } \\
\text { ensino médio incompleto }\end{array}$ & $0,74(0,73-0,75)$ ฯ & $0,75(0,74-0,77)$ ฯ & $0,72(0,71-0,72)$ ฯ & $0,72(0,72-0,73)$ ๆ \\
\hline $\begin{array}{l}\text { Ensino médio completo/ } \\
\text { ensino superior incompleto }\end{array}$ & $0,56(0,56-0,57)$ ฯ & $0,59(0,58-0,61)$ ฯ & $0,53(0,52-0,53) \pi$ & $0,53(0,53-0,54) \pi$ \\
\hline Ensino superior completo & $0,45(0,44-0,45)$ ๆ & $0,53(0,51-0,56) \pi$ & $0,44(0,44-0,45) \rrbracket$ & $0,52(0,50-0,53) \pi$ \\
\hline Não informada & $0,58(0,52-0,63) \pi$ & $0,79(0,67-0,94)$ & $0,59(0,56-0,62)$ ฯ & $0,69(0,63-0,77) \pi$ \\
\hline
\end{tabular}


Tabela 4 - Regressão binomial negativa para estimar a associação entre cor/raça e parturição, estratificada por região e situação urbana/rural, ajustada para idade, escolaridade, total de cômodos do domicílio e número de moradores do domicílio. Regiões Norte e Nordeste - Brasil - 2010 (cont.)

\begin{tabular}{|c|c|c|c|c|}
\hline \multirow{3}{*}{ Variável } & \multicolumn{4}{|c|}{$\begin{array}{l}\text { Coeficiente de regressão para parturição } \\
\text { (intervalo de confiança de } 95 \% \text { ) }\end{array}$} \\
\hline & \multicolumn{2}{|c|}{ Norte } & \multicolumn{2}{|c|}{ Nordeste } \\
\hline & Urbana & Rural & Urbana & Rural \\
\hline \multicolumn{5}{|c|}{ Idade (anos) } \\
\hline $10-14$ & $0,16(0,11-0,22)$ ฯ & $0,11(0,07-0,16)$ ฯ & $0,17(0,13-0,22)$ ฯ & $0,15(0,11-0,19) 9$ \\
\hline $15-19$ & $0,17(0,16-0,18) \pi$ & $0,18(0,17-0,19)$ ฯ & $0,18(0,17-0,18)$ ฯ & $0,18(0,17-0,18)$ \\
\hline $20-29$ & $0,61(0,61-0,62)$ ฯ & $0,60(0,59-0,61)$ ฯ & $0,62(0,61-0,62)$ ฯ & $0,60(0,59-0,60) 9$ \\
\hline $30-39$ & $1,00(-)$ & $1,00(-)$ & $1,00(-)$ & $1,00(-)$ \\
\hline $40-49$ & $1,26(1,24-1,27)$ I & $1,29(1,27-1,32)$ I & $1,28(1,27-1,29) \mathbb{\pi}$ & $1,40(1,38-1,41) 9$ \\
\hline $50-59$ & $1,58(1,56-1,60)$ ฯ & $1,61(1,58-1,64)$ ฯ & $1,72(1,70-1,73) \pi$ & $1,92(1,91-1,94) q$ \\
\hline $60+$ & $2,13(2,10-2,16) \pi$ & $1,91(1,87-1,94)$ I & $2,54(2,52-2,56) \pi$ & $2,50(2,47-2,52) 9$ \\
\hline \multicolumn{5}{|c|}{ Número de cômodos no domicílio } \\
\hline $1+$ & $1,00(1,00-1,00)$ & $1,00(1,00-1,00)$ & $1,00(1,00-1,00) \pi$ & $1,00(1,00-1,00)$ \\
\hline \multicolumn{5}{|c|}{ Número de moradores no domicílio } \\
\hline $1+$ & $1,00(1,00-1,00)$ & $1,00(1,00-1,00)$ & $1,00(1,00-1,00)$ & $1,00(1,00-1,00)$ \\
\hline
\end{tabular}

*A categoria de referência do termo de interação foi constituída por mulheres de cor/raça branca, que responderam diretamente aos recenseadores.

I Associações estatisticamente significativas ( $p<0,05$ ), conforme o teste de heterogeneidade de Wald. Apenas coeficientes (razões de incidência) para a contagem de filhos tidos são apresentados na tabela.

Fonte: estimativas produzidas pelos próprios autores com base nos dados analisados. 
Gráfico 1 - Médias de parturição (e seus intervalos de confiança de 95\%) conforme cor/raça e tipo de respondente, ajustadas para idade, escolaridade, total de cômodos do domicílio e número de moradores do domicílio. Região Nordeste - Brasil - 2010
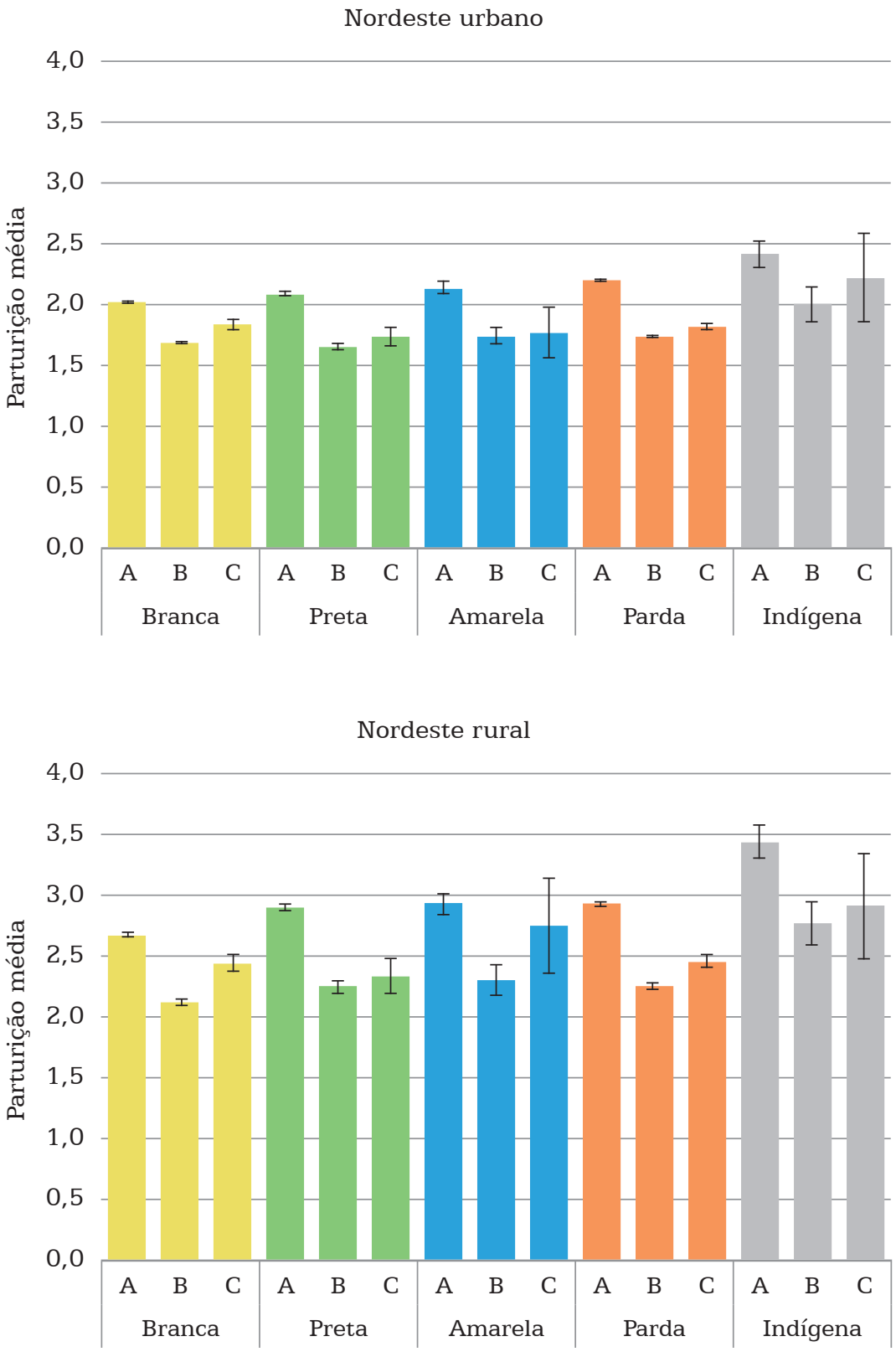

Obs.: A = informação fornecida pela própria mulher, $\mathrm{B}=$ informação provida por outro morador, C = informação dada por não morador.

Fonte: estimativas produzidas pelos próprios autores com base nos dados analisados. 
Gráfico 2 - Médias de parturição (e seus intervalos de confiança de 95\%) conforme cor/raça e tipo de respondente, ajustadas para idade, escolaridade, total de cômodos do domicílio e número de moradores do domicílio. Região Norte - Brasil - 2010
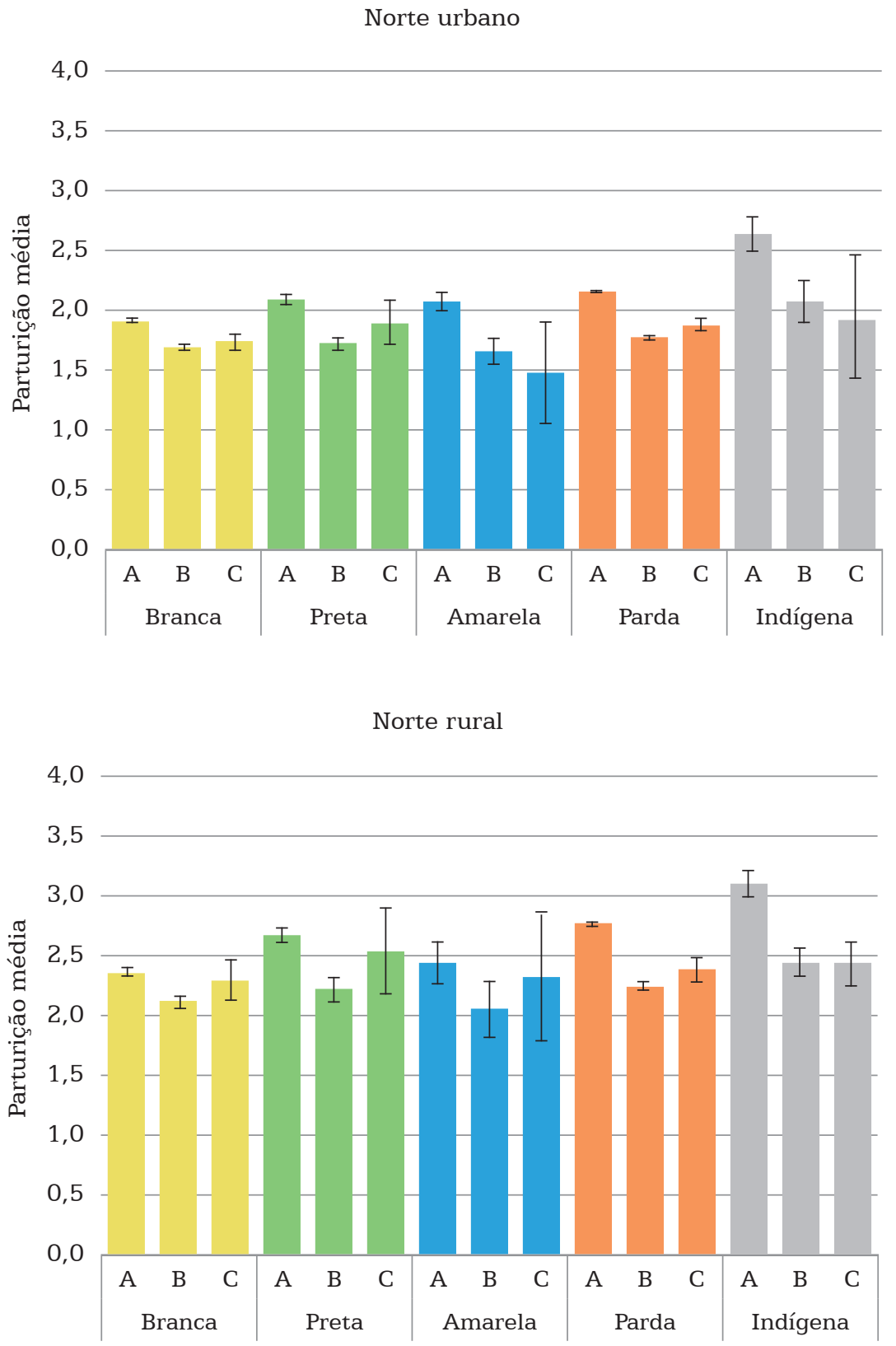

Obs.: $\mathrm{A}=$ informação fornecida pela própria pessoa; $\mathrm{B}=$ informação provida por outro morador; $\mathrm{C}=$ informação dada por não morador.

Fonte: estimativas produzidas pelos próprios autores com base nos dados analisados. 


\section{Interpretação e implicações dos achados}

Em meio à ainda limitada, mas crescente, literatura sobre demografia dos povos indígenas no Brasil baseada em dados censitários (Azevedo, 2011; IBGE, 2005, 2012; Pagliaro, Azevedo G Santos, 2005; Perz et al., 2008; Santos G Teixeira, 2011), pouca atenção tem sido dada ao fato de que sociedades culturalmente diferenciadas podem apresentar características, incluindo filiação linguística, que potencialmente afetam os padrões de resposta dados aos recenseadores. Os resultados deste estudo demonstraram que as mulheres indígenas, especialmente as da região Norte, responderam diretamente aos entrevistadores do censo em uma percentagem expressivamente menor do que aquelas de outras categorias de cor/raça. Um achado que merece destaque foi que 12,7\% das mulheres indígenas do Norte tiveram suas informações fornecidas ao censo por um não morador de seus domicílios, frequência bastante acima daquela observada para outros grupos de cor/raça da mesma região, a qual variou entre 2,5\% (amarelas) e 4,0\% (brancas). É igualmente revelador que, no caso das mulheres do Nordeste, tais diferenças não foram detectadas.

Observou-se também que as diferenças mais expressivas na parturição média estão relacionadas ao tipo de respondente. Quando a contagem de filhos não é informada diretamente pela mãe, mas por respondentes proxy (tanto um morador quanto um não morador do domicílio), os valores médios de parturição tendem a ser consideravelmente mais baixos. Esse não é apenas o caso das mulheres indígenas, mas também o dos demais grupos de cor/raça nas duas regiões abordadas, tanto em áreas urbanas quanto rurais. O maior percentual de mulheres indígenas cujas informações foram providas por um não morador no Norte $(12,7 \%)$ sugeriu que a relação entre parturição e tipo de respondente pode ser diferente nas duas regiões investigadas. Entretanto, após controlar para um conjunto de covariáveis no contexto de modelos de regressão, constatou-se que a subestimação da parturição de mulheres indígenas foi semelhante no Norte e no Nordeste, tanto entre outros moradores quanto entre não moradores dos domicílios. Deve-se salientar, contudo, que as comparações de parturição, levando em consideração situação urbana/rural e tipo de respondente, indicam que a subestimação da parturição foi particularmente pronunciada entre as indígenas do Norte rural (16\% menor em relação às mulheres brancas que responderam diretamente aos recenseadores).

Independentemente da cor/raça, a subestimação da parturição possivelmente deriva do fato de que respondentes proxynão estão em condições de informar precisamente o número de filhos, o que também se aplicaria a outros dados relativos à história reprodutiva das mulheres. Por exemplo, respondentes proxy podem desconhecer filhos que já vieram a falecer ou que não coabitam o mesmo domicílio da mãe. Quando a informação não é fornecida diretamente pela mulher, tais casos 
podem ser omitidos da contagem. O fato de que os povos indígenas no Brasil apresentam maiores taxas de mortalidade infantil pode exercer um impacto adicional sobre a contagem de filhos informada por respondentes proxy, na medida em que estes tendem a desconhecer suas histórias reprodutivas (Cardoso, Santos G Coimbra, 2005; IBGE, 2005). A combinação dessas duas dimensões - menores frequências de mulheres indígenas relatando a quantidade de filhos diretamente aos entrevistadores e tendência a fornecer dados menos precisos por parte dos respondentes proxy - culminou em um efeito sinérgico, o qual pode contribuir para a compreensão da subestimação ainda mais acentuada da parturição das mulheres indígenas, especialmente na região Norte.

Quais fatores poderiam explicar a menor frequência de respostas dadas diretamente aos entrevistadores entre as mulheres indígenas da região Norte? É provável que aspectos socioculturais exerçam um papel central nesse caso. Relativamente a outras regiões brasileiras, as sociedades indígenas que vivem no Norte puderam preservar mais não somente as suas línguas nativas, mas também seus territórios tradicionais (Hemming, 1987; Ricardo G Ricardo, 2011). A razão para tais acontecimentos é histórica. A região Norte foi a última a experimentar colonização em larga escala, particularmente dos anos 1960 em diante. Em alguma medida, apesar das altas taxas de mortalidade e violência, no período mais recente, movimentos indígenas têm conseguido assegurar alguns direitos às sociedades indígenas do Norte, não se observando o mesmo para outras regiões do país.

A colonização do Nordeste por não indígenas teve início no século XVI, o que significa que as sociedades indígenas nessa área foram as primeiras a experimentar os impactos devastadores da chegada dos europeus, que acarretaram redução massiva das populações, perda de territórios e de línguas (Hemming, 1987; Oliveira-Filho, 2011; Ricardo G Ricardo, 2011). Dados do Censo Demográfico 2010 demonstram como algumas características culturais e socioeconômicas das sociedades indígenas atuais refletem os efeitos de longa duração de quinhentos anos de colonização por não indígenas. Enquanto 26,2\% dos declarados indígenas com 5 anos de idade ou mais na região Norte não falavam português, tal estimativa foi de apenas 3,2\% no Nordeste (IBGE, 2012). Tendo esse complexo cenário sociopolítico e histórico como pano de fundo, o fato de que o censo é conduzido em português pode influenciar padrões de coleta de dados em algumas circunstâncias, tais como aquelas apresentadas por determinados grupos indígenas, entre os quais parte ou a totalidade da população não utiliza o português em sua comunicação cotidiana.

No âmbito deste estudo, a variável relativa ao tipo de respondente foi central. Um aspecto relevante diz respeito à identidade dos não moradores que, tal como vimos para a região Norte em particular, exerceu um papel importante no fornecimento de informações das mulheres indígenas. De acordo com o IBGE, principalmente em centros urbanos do Brasil, o não morador é frequentemente uma trabalhadora doméstica, 
que está no domicílio no momento da visita do entrevistador (IBGE, 2013). No caso dos povos indígenas, é improvável que esse também seja o caso, de modo que não moradores geralmente incluem profissionais da saúde ou professores que trabalham e vivem nas comunidades e que também possuem ancestralidade indígena, na maior parte dos casos. Outras pesquisas de abrangência nacional, tal como o Primeiro Inquérito Nacional de Saúde e Nutrição Indígena, realizado em 2008 (Coimbra et al., 2013), documentaram o importante papel exercido por esses profissionais no que se refere às dinâmicas e ao estabelecimento de vínculos com as comunidades, incluindo sua contribuição como intérpretes durante a realização das entrevistas. Ainda que residam e trabalhem nesses locais e estejam, portanto, familiarizados com características culturais e, eventualmente, com a língua nativa, profissionais da saúde ou professores não estão necessariamente a par da história reprodutiva das mulheres a ponto de fornecer informações tão precisas quanto elas próprias.

Embora este capítulo tenha enfatizado um conjunto específico de variáveis relacionadas com a parturição, os aspectos levantados têm implicações para uma ampla sorte de outras condições de interesse. Da mesma forma que a parturição de mulheres indígenas pode ser afetada pelo tipo de respondente e pela frequência com que cada um deles provê informação aos recenseadores, as estimativas de diversos outros parâmetros demográficos, desde coeficientes de mortalidade a condições socioeconômicas, podem ser igualmente influenciadas por processos semelhantes. Em íntima relação com a parturição, as estimativas de fecundidade constituem um bom exemplo adicional. Análises baseadas no Censo Demográfico 2010 (IBGE, 2010b) mostraram altas taxas de fecundidade total (TFT) para mulheres indígenas: 2,90 no Sudeste, 3,01 no Nordeste, 3,74 no Sul, 4,30 no Centro-Oeste e 4,92 no Norte, com uma estimativa global para o país de 3,88 (IBGE, 2012). Tal como tem sido sugerido em diversas outras investigações voltadas para a caracterização dos padrões de fecundidade no Brasil (Carvalho G Brito, 2005; Goldani, 2009; Potter et al., 2010), essas diferenças inter-regionais na fecundidade indígena possivelmente também derivam de uma complexa interação entre fatores comportamentais e sociodemográficos, incluindo escolaridade, participação da mulher no mercado de trabalho e acesso a métodos contraceptivos, entre outros. Pode-se argumentar que, se o tipo de respondente fosse levado em consideração, as TFTs para mulheres indígenas do Norte seriam ainda mais altas, afetando as comparações inter-regionais para esse segmento da população. Em uma dimensão mais ampla, as análises dos padrões demográficos de populações brasileiras (indígenas e não indígenas), incluindo os importantes tópicos da transição nos níveis de mortalidade e fecundidade, deveriam levar em consideração que as estimativas são potencialmente afetadas por fatores não estritamente demográficos. 


\section{Considerações finais}

Análises das características demográficas dos povos indígenas baseadas em dados censitários têm se tornado cada vez mais importantes nos debates sobre saúde, educação e outras desigualdades socioeconômicas em todo o mundo (Axelsson G Sköld, 2011; Axelsson et., 2011; Kertzer G Arel, 2002; Morning, 2008; Paho, 2006; UN, 2009). Contudo, tal como Axelsson e Sköld (2011: 5) têm argumentado em relação às limitações dos inquéritos de larga escala não especificamente planejados para coletar dados em meio a povos indígenas, "censos conduzidos por agências externas, dados administrativos e de inquéritos são frequentemente imprecisos, quando se trata de representar as estruturas sociais indígenas". Ao mesmo tempo que é fundamental expandir a coleta de dados demográficos de populações indígenas, não se pode negligenciar o fato de que a qualidade dos dados pode ser afetada por condições específicas nas quais a coleta transcorre. O estudo de caso de populações indígenas do Brasil aqui apresentado proporciona evidência contundente de que os padrões de resposta de mulheres indígenas aos entrevistadores do censo podem influenciar a estimação de parâmetros demográficos. Esse fenômeno também foi observado para mulheres não indígenas, porém, não com a mesma intensidade.

Algumas iniciativas recentes na América Latina, tal como a inclusão de perguntas sobre línguas indígenas nos questionários dos censos, são avanços bem-vindos que podem contribuir para melhorar a caracterização da demografia indígena (Del Popolo, 2008; Del Popolo et al., 2011; Frutos, Sanabria G Servin, 2004). Enquanto rotinas específicas para coleta de dados em populações culturalmente diferenciadas, tais como os povos indígenas, não forem implementadas de modo mais amplo, tanto na América Latina quanto em outras regiões do mundo, mais atenção deve ser dada aos diferenciais demográficos que podem resultar dos processos de coleta de dados. É desnecessário enfatizar que tais diferenciais podem influenciar a definição e a implementação de políticas públicas de saúde destinadas ao combate da marginalização socioeconômica que caracteriza todos os povos indígenas ao redor do mundo. A implementação de alternativas metodológicas nos censos brasileiros, incluindo a seleção e o treinamento de entrevistadores fluentes nas línguas nativas e familiarizados com suas respectivas culturas, pode ser útil na melhoria da qualidade da coleta de dados. Tendo em vista que os diferenciais de parturição, conforme o tipo de respondente, foram observados em todos os grupos de cor/raça, futuras investigações sobre estimativas desse parâmetro e os fatores que o afetam devem ser realizadas em todas regiões do país. 
* Este capítulo é uma tradução, com adaptações, de artigo previamente publicado por Santos e colaboradores (2015). Foi reproduzido aqui com a devida autorização.

1 Os dados dos censos nacionais estão disponibilizados em páginas eletrônicas de domínio público e não houve necessidade de aprovação prévia do protocolo do presente estudo por um comitê de ética, de acordo com a resolução de ética para pesquisa com seres humanos no Brasil utilizando dados secundários (Conselho Nacional de Saúde, resolução n. 466/2012).

\section{Referências}

AXELSSON, P. G SKÖLD, P. Indigenous peoples and demography. In: AXELSSON, P. G SKÖLD, P. (Eds.). Indigenous Peoples and Demography: the complex relation between identity and statistics. New York: Berghahn Books, 2011.

AXELSSON, P. et al. Epilogue: from indigenous demographics to an indigenous demography. In: AXELSSON, P. G SKÖLD, P. (Eds.). Indigenous Peoples and Demography: the complex relation between identity and statistics. New York: Berghahn Books, 2011.

AZEVEDO, M. M. O Censo 2010 e os povos indígenas. In: RICARDO, C. A. G RICARDO, F. (Orgs.). Povos Indígenas do Brasil 2006/2010. São Paulo: Instituto Socioambiental, 2011.

CARDOSO, A. M.; SANTOS, R. V. G COIMBRA, C. E. A. Mortalidade infantil segundo raça/cor no Brasil: o que dizem os sistemas nacionais de informação? Cadernos de Saúde Pública, 21(5): 1.602-1.608, 2005.

CARVAlHO, J. A. M. G BRITO, F. A demografia brasileira e o declínio da fecundidade no Brasil: contribuições, equívocos e silêncios. Revista Brasileira de Estudos Populacionais, 22(2): 351-369, 2005.

COIMBRA JR., C. E. A. G SANTOS, R. V. Emerging health needs and epidemiologic research in indigenous peoples in Brazil. In: SALZANO, F. M. G HURTADO, M. (Eds.). Lost Paradise and the Ethics of Research and Publication. Oxford: Oxford University Press, 2004.

COIMBRA JR., C. E. et al. The first national survey of indigenous people's health and nutrition in Brazil: rationale, methodology, and overview of results. BMC Public Health, 13: 52, 2013. Disponível em: <www.biomedcentral.com/1471-2458/13/52> . Acesso em: 10 set. 2014.

DEL POPOLO, F. Los Pueblos Indígenas y Afrodescendientes en las Fuentes de Datos: experiencias en América Latina. Santiago: Comisión Económica para América Latina y el Caribe, 2008. 
DEL POPOLO, F. et al. El Derecho de la Información de los Pueblos Indígenas y Afrodescendientes: obligaciones urgentes en América Latina. Santiago: Centro Latinoamericano y Caribeño de Demografia, 2011.

FRUTOS, N. D.; SANABRIA, C. L. F. G SERVIN, Z. C. S. Atlas de las Comunidades Indígenas en el Paraguay. Assunción: Dirección General de Estadística, Encuestas y Censos, 2004.

GOLDANI, A. M. What Will Happen to Brazilian Fertility? New York: United Nations, 2009.

GONÇALVES, J. M. M. IBGE: um retrato histórico. Rio de Janeiro: IBGE, 1995.

HEMMING, J. The Amazon Frontier: the defeat of the Brazilian indians. London: MacMillan Press, 1987.

INCAYAWAR, M. G MALDONADO-BOUCHARD, S. The forsaken mental health of the indigenous peoples: a moral case of outrageous exclusion in Latin America. BMC International Health and Human Rights, 9: 27, 2009. Disponível em: <www.biomedcentral.com/1472698X/9/27>. Acesso em: 17 set. 2014.

INSTITUTO BRASILEIRO DE GEOGRAFIA E ESTATÍSTICA (IBGE). Tendências Demográficas: uma análise dos indígenas com base nos resultados da amostra dos Censos Demográficos 1991 e 2000. Rio de Janeiro: IBGE, 2005.

INSTITUTO BRASILEIRO DE GEOGRAFIA E ESTATÍSTICA (IBGE). Estatísticas do Século $X X$. Rio de Janeiro: IBGE, 2006.

INSTITUTO BRASILEIRO DE GEOGRAFIA E ESTATÍSTICA (IBGE). Censo Demográfico 2010: manual do recenseador. Rio de Janeiro: IBGE, 2010a.

INSTITUTO BRASILEIRO DE GEOGRAFIA E ESTATístiCA (IBGE). Censo Demográfico 2010: nupcialidade, fecundidade e migração - resultados da amostra. Rio de Janeiro: IBGE, 2010b.

INSTITUTO BRASILEIRO DE GEOGRAFIA E ESTATÍSTICA (IBGE). Censo Demográfico 2010: características gerais dos indígenas - resultados do universo. Rio de Janeiro: IBGE, 2012.

INSTITUTO BRASILEIRO DE GEOGRAFIA E ESTATÍstiCA (IBGE). Metodologia do Censo Demográfico 2010. Rio de Janeiro: IBGE, 2013.

KERTZER, D. G AREL, D. Census and Identity: the politics of race, ethnicy and language in national censuses. Cambridge: Cambridge University Press, 2002.

McSWEENEY, K. G ARPS, S. A "demographic turnaround": the rapid growth of the indigenous populations in lowland Latin America. Latin American Research Review, 40(1): 3-29, 2005.

MONTENEGRO, R. A. G STEPHENS, C. Indigenous health in Latin America and the Caribbean. The Lancet, 367(9.525): 1.859-1.869, 2006.

MORNING, A. Ethnic classification in global perspective: a cross-national survey of the 2000 census round. Population Research and Policy Review, 27(2): 239-272, 2008. 
NOBLES, M. Shades of Citizenship: race and the census in modern politics. Stanford: Stanford University Press, 2000.

OLIVEIRA-FILHO, J. P. A Presença Indígena no Nordeste: processos de territorialização, modos de reconhecimento e regimes de memória. Rio de Janeiro: Contra Capa, 2011.

PAGLIARO, H.; AZEVEDO, M. M. G SANTOS, R. V. Demografia dos Povos Indígenas no Brasil. Rio de Janeiro: Editora Fiocruz, Abep, 2005.

PAN AMERICAN HEALTH ORGANIZATION (PAHO). Health of the Indigenous Peoples in the Americas. Washington: Pan American Health Organization, 2006.

PERZ, S. G.; WARREN, J. G KENNEDY, D. P. Contributions of racial-ethnic reclassification and demographic processes to indigenous population resurgence: the case of Brazil. Latin American Research Review, 43(2): 7-33, 2008.

POTTER, J. E. et al. Mapping the timing, pace, and scale of the fertility transition in Brazil. Population and Development Review, 36(2): 283-307, 2010.

RICARDO, C. A. G RICARDO, F. Povos Indígenas no Brasil 2006/2010. São Paulo: Instituto Socioambiental, 2011.

SANTOS, R. V. G TEIXEIRA, P. O "indígena" que emerge do Censo Demográfico de 2010. Cadernos de Saúde Pública, 27(6): 1.048, 2011.

SANTOS, R. V. et al. Parity of indigenous and non-indigenous women in Brazil: does the reported number of children born depend upon who answers National Census questions? Plos One, 10(4), 2015. Disponível em: < https://doi.org/10.1371/journal.pone.0123826>. Acesso em: 10 mar. 2019.

UNITED NATIONS (UN). State of the World's Indigenous Peoples. New York: United Nations, 2009. 